\title{
Nitric oxide vs insulin secretion, action and clearance
}

\author{
Olga Kruszelnicka
}

Received: 9 September 2013 / Accepted: 23 September 2013 /Published online: 18 October 2013

(C) Springer-Verlag Berlin Heidelberg 2013

Keywords Asymmetric dimethylarginine · Insulin clearance · Insulin resistance $\cdot$ Insulin secretion $\cdot$ Nitric oxide

$\begin{array}{ll}\text { Abbreviations } \\ \text { ADMA } & \text { Asymmetric dimethylarginine } \\ \text { IR } & \text { Insulin resistance } \\ \text { L-NAME } & N^{\mathrm{G}} \text {-nitro-L-arginine methylester } \\ \text { TXNIP } & \text { Thioredoxin-interacting protein }\end{array}$

To the Editor: In their Diabetologia article, Natali and colleagues [1] reported a decrease in glucose tolerance in response to acute inhibition of $\mathrm{NO}$ generation by $N^{\mathrm{G}}$-nitro-L-arginine methylester (L-NAME), which resulted from a $40 \%$ drop in beta cell glucose sensitivity and doubled insulin clearance, whereas peripheral insulin sensitivity did not change. Admittedly, whether conclusions from an acute experiment can apply to a long-term impairment of NO bioavailability remains disputable. Moreover, mice with genetic disruption of endothelial NO synthase, used as a model of chronic NO deficiency, exhibit insulin resistance (IR) in the liver and peripheral tissues [2]. Nevertheless, it can be speculated that depressed insulin secretion and enhanced insulin degradation, when superimposed on concomitant IR, might further impair glucose tolerance and accelerate the development of type 2 diabetes mellitus under conditions associated with reduced $\mathrm{NO}$ bioavailability.

Since the dose of L-NAME shown to decrease insulinaemia also induced endothelial dysfunction [1], these findings support the notion of impaired endothelial function, largely mediated by decreased NO bioavailability, as an independent

O. Kruszelnicka $(\bowtie)$

Department of Coronary Artery Disease, The John Paul II Hospital in Kraków, 80 Prądnicka Street, 31-202 Kraków, Poland

e-mail: olga.kruszelnicka@onet.pl predictor of new-onset type 2 diabetes [3, 4]. Furthermore, the report by Natali et al [1] appears to link these observations to relatively reduced insulin concentrations (i.e. lower than would be expected for the degree of IR), in contrast to other investigators who have suggested that endothelial dysfunction in skeletal muscle - via blunted muscular perfusion, reduced capillary recruitment and decreased insulin delivery into the interstitium - could lead to IR thus predisposing individuals to type 2 diabetes [3, 4].

The results by Natali et al [1] also explain the previously reported association between the endogenous NO synthesis inhibitor asymmetric dimethylarginine (ADMA) and the risk of future deterioration of glucose tolerance [5, 6]. Following the report of this association by Mittermayer et al [5] in 77 women with previous gestational diabetes, we have identified the same relationship in 80 non-diabetic men with stable angina who were followed for a 4.5 year period [6]. Mittermayer et al [5] observed an independent association of declining glucose tolerance during almost 3 years with higher ADMA but not HOMA-IR 14-16 weeks after delivery, whereas in our hands ADMA and HOMA-IR independently predicted deteriorating glucose tolerance [6]. Moreover, in both reports ADMA and HOMA-IR were unrelated [5, 6]. Thus, on the basis of the findings by Natali et al [1], the predictive ability of ADMA in these studies $[5,6]$ might hypothetically be attributable not to concomitant IR but possibly to accompanying relative insulin deficiency, inasmuch as the long-term effects of ADMA are similar to those of shortterm NO inhibition. Nevertheless, this concept is challenged by the recent concerns raised by Reaven [7] with regard to using HOMA-IR as an index of IR when altered insulin clearance is the major determinant of the change in fasting insulinaemia.

Intriguingly, the effects of $\mathrm{NO}$ on both insulin catabolism and beta cell glucose sensitivity are at least partially mediated by the ability of NO to exert biological effects independently 
of its classical second messenger, cGMP. Accordingly, NO inhibits the insulin-degrading enzyme by $S$-nitrosylation [8], i.e. the reversible covalent modification of thiol groups on cysteine residues. Additionally, in beta cells, elevations in NO production occur within $20 \mathrm{~s}$ of glucose stimulation, and $S$-nitrosylation of glucokinase at cysteine-371 or syntaxin 4 at cysteine-141 may facilitate glucose-stimulated insulin secretion [9].

In addition, NO cGMP independently represses the transcription of thioredoxin-interacting protein (TXNIP), which not only enables antioxidative actions of thioredoxins, but also stimulates thioredoxin-dependent protein denitrosylation, thus providing a negative feedback mechanism protecting against excessive protein $S$-nitrosylation and nitrosative stress [10]. Shaked et al [11] demonstrated that the NO-dependent TXNIP inhibition contributed to the protective effect of insulin against glucose-induced beta cell apoptosis. Finally, Wang et al [12] recently reported the ability of NO to stimulate transendothelial insulin transport by enhancing protein $S$-nitrosylation, which could be abolished by knockout of TXNIP.

Hence, protein $S$-nitrosylation can influence insulin secretion, action and clearance. Under conditions associated with endothelial dysfunction and impaired NO bioavailability, protein $S$-nitrosylation, generally assumed to be a non-enzymatic process, might be differentially affected from cGMP-dependent events modulating insulin secretion and signalling. The clinical relevance of this hypothetical shift in NO signal transduction remains to be determined. Further studies are warranted to elucidate as yet unravelled interactions between endothelial dysfunction and insulin metabolism and activity.

Duality of interest The author declares that there is no duality of interest associated with this manuscript.

Contribution statement The author was the sole contributor to this paper.

\section{References}

1. Natali A, Ribeiro R, Baldi S et al (2013) Systemic inhibition of nitric oxide synthesis in non-diabetic individuals produces a significant deterioration in glucose tolerance by increasing insulin clearance and inhibiting insulin secretion. Diabetologia 56:1183-1191

2. Shankar RR, Wu Y, Shen HQ, Zhu JS, Baron AD (2000) Mice with gene disruption of both endothelial and neuronal nitric oxide synthase exhibit insulin resistance. Diabetes 49:684-687

3. Meigs JB, Hu FB, Rifai N, Manson JE (2004) Biomarkers of endothelial dysfunction and risk of type 2 diabetes mellitus. JAMA 291: 1978-1986

4. Perticone F, Maio R, Sciacqua A et al (2008) Endothelial dysfunction and $\mathrm{C}$-reactive protein are risk factors for diabetes in essential hypertension. Diabetes 57:167-171

5. Mittermayer F, Kautzky-Willer A, Winzer C et al (2007) Elevated concentrations of asymmetric dimethylarginine are associated with deterioration of glucose tolerance in women with previous gestational diabetes mellitus. J Intern Med 261:392-398

6. Surdacki A, Kruszelnicka O, Rakowski T, Jaźwińska-Kozuba A, Dubiel JS (2013) Asymmetric dimethylarginine predicts decline of glucose tolerance in men with stable coronary artery disease: a 4.5-year follow-up study. Cardiovasc Diabetol 12:64

7. Reaven GM (2013) What do we learn from measurements of HOMA-IR? Diabetologia 56:1867-1868

8. Cordes CM, Bennett RG, Siford GL, Hamel FG (2009) Nitric oxide inhibits insulin-degrading enzyme activity and function through $S$-nitrosylation. Biochem Pharmacol 77:1064-1073

9. Wiseman DA, Thurmond DC (2012) The good and bad effects of cysteine S-nitrosylation and tyrosine nitration upon insulin exocytosis: a balancing act. Curr Diabetes Rev 8:303-315

10. Forrester MT, Seth D, Hausladen A et al (2009) Thioredoxininteracting protein (Txnip) is a feedback regulator of $S$-nitrosylation. J Biol Chem 284:36160-36166

11. Shaked M, Ketzinel-Gilad M, Ariav Y, Cerasi E, Kaiser N, Leibowitz G (2009) Insulin counteracts glucotoxic effects by suppressing thioredoxin-interacting protein production in INS-1E beta cells and in Psammomys obesus pancreatic islets. Diabetologia 52: 636-644

12. Wang H, Wang AX, Aylor K, Barrett EJ (2013) Nitric oxide directly promotes vascular endothelial insulin transport. Diabetes. doi:10.2337/db13-0627 